\title{
ESTUDO ELETROQUÍMICO DOS FLAVONOIDES: QUERCETINA E NARINGENINA
}

\author{
Grazielle Santos Castro \\ Mateus Rocha \\ Edgard Antonio Ferreira \\ Anamaria Dias Pereira Alexiou \\ Jairo José Pedrotti \\ Universidade Presbiteriana Mackenzie (UPM)
}

\begin{abstract}
Resumo
Este artigo avalia o comportamento eletroquímico dos flavonoides quercetina e naringenina em soluçóes com diferentes valores de $\mathrm{pH}$. O estudo foi realizado com eletrodos de trabalho de carbono vítreo e carbono vítreo modificado com filme de óxido de grafeno reduzido (rGO). Em solução tampão Britton-Robinson, com pH de 6,41, a quercetina apresenta três picos de oxidação sobre o eletrodo de carbono vítreo que se intensificam com a maior velocidade de varredura. As medidas realizadas por voltametria cíclica com flavonoides feitas com o eletrodo modificado (rGO/GCE) mostraram maior intensidade do sinal de corrente. Ensaios voltamétricos realizados com eletrodo de rGO mostraram que, na faixa de potencial que se estende de 0,0 a $0,30 \mathrm{~V}$ vs. $\mathrm{Ag} / \mathrm{AgCl}$, o sensor permite a determinação seletiva de quercetina na presença de naringerina em extrato da planta Ludwigia repens.
\end{abstract}

Palavras-chave: Eletrodo modificado. Naringenina. Óxido de grafeno reduzido. Quercetina. 


\section{INTRODUÇÁO}

Os flavonoides são substâncias polifenólicas encontradas em diversas plantas, frutas e sementes. Esses compostos têm atraído considerável atenção de pesquisadores por suas propriedades antioxidantes e por suas notáveis atividades biológicas e farmacológicas (MALEKIA; CRESPO; CABANILLAS, 2019; MUTHA; TATIYA; SURANA, 2021). A atividade antioxidante de uma substância é atribuída às características de neutralização (ou sequestro) de radicais livres e quelação de metais de transição, agindo tanto na etapa de iniciação quanto na propagação do processo oxidativo. Os intermediários formados pela ação de antioxidantes fenólicos são relativamente estáveis, devido à ressonância do anel aromático presente na estrutura dessas substâncias (CHAVES et al., 2007; GUAN et al. 2021). A quercetina (3,3',5,7,4'-pentahidroxiflavona) e a naringenina (4',5,7'-tri-hidroxi flavona) são flavonoides amplamente encontrados em frutas e vegetais, com maior predominância em maçấs e cerejas (quercetina) e em frutas cítricas e tomates (naringenina). A estrutura química dos flavonoides é formada por um sistema de três anéis, incluindo dois aromáticos ( $\mathrm{A} \mathrm{e} \mathrm{B)} \mathrm{ligados} \mathrm{por} \mathrm{três} \mathrm{áto-}$ mos de carbono formando um anel heterocíclico oxigenado (anel C), representada na Figura 1.
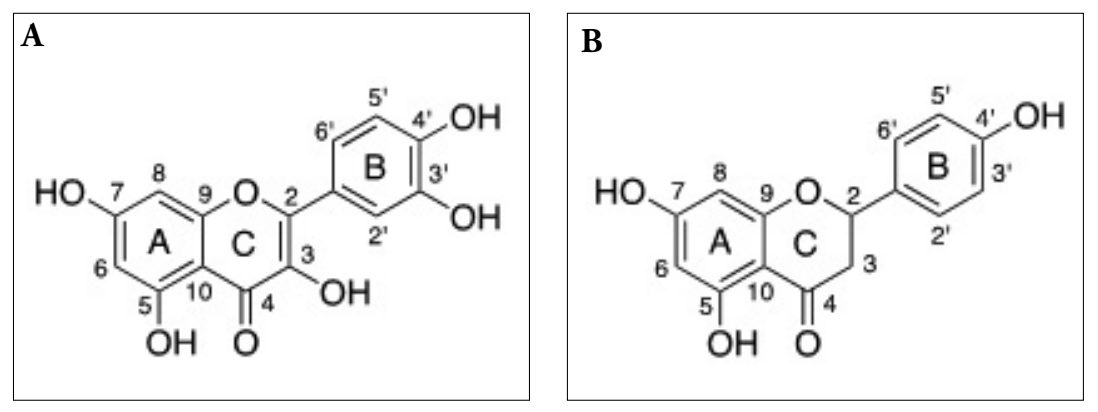

Figura 1 Representação da estrutura molecular da quercetina (A) e naringenina (B).

Fonte: Kosanović et al. (2010) e Mülazimoğlu, Özkan e Solak (2011).

As características antioxidante e antirradicalar estão diretamente ligadas à estrutura molecular, que são alteradas conforme a adição e a posição de determinados grupos funcionais. A presença de uma hidroxila na posição 3 (anel C), bem como de uma ligação dupla entre os carbonos 2 e 3 (anel C), de uma carbonila no carbono 4 e poli-hidroxilação dos anéis $\mathrm{A}$ e $\mathrm{B}$ são fatores determinantes para a atividade biológica dos 
compostos fenólicos, e, quanto maior a quantidade de hidroxilas livres, maior será a atividade antirradicalar ou redutora do flavonoide (SÁ, 2013).

Para a caracterização detalhada desses compostos antioxidantes, técnicas eletroquímicas podem ser aplicadas com sucesso, pois fornecem parâmetros físico-químicos capazes de mostrar não apenas o potencial redox, mas também o número de elétrons envolvidos na reação $(\mathrm{n})$, os mecanismos de transferência de elétrons na superfície do eletrodo e a influência de prótons (REIS et al., 2009).

Os métodos eletroquímicos fazem uso de propriedades elétricas mensuráveis (como corrente elétrica, diferenças de potencial, acúmulo interfacial de carga, entre outros) a partir de fenômenos, nos quais uma espécie redox interage física e/ou quimicamente com os demais componentes, ou interfaces, do meio. Tais interaçóes são observadas quando se aplicam perturbaçóes controladas ao sistema; por exemplo, uma diferença de potencial entre eletrodos de uma cela eletroquímica (PACHECO et al., 2013).

O grafeno é formado por uma monocamada plana de átomos de carbono com hibridização $\mathrm{sp}^{2}$ e estrutura bidimensional. Desde o seu isolamento, em 2004, esse material tem despertado interesse crescente da comunidade científica devido às suas propriedades físicas fascinantes, como a área superficial extremamente elevada (2600 $\mathrm{m}^{2} / \mathrm{g}$ ), a transparência à luz visível $(97,7 \%)$, a excelente resistência mecânica, a boa condutividade térmica e a elevada condutividade elétrica (NOVOSELOV et al., 2004; CLASS FOR PHYSICS OF THE ROYAL SWEDISH ACADEMY OF SCIENCES, 2010; LIU et al., 2011). O óxido de grafeno (GO) é uma forma oxidada do grafeno. Esse material bidimensional é caracterizado pela abundante quantidade de grupos funcionais oxigenados na sua estrutura. A presença de hidroxilas e grupamento epóxi no plano basal do GO torna o material hidrofílico, facilitando sua dispersão em água, em estruturas de uma ou poucas camadas. Adicionalmente, com o rompimento de ligaçóes $\mathrm{sp}^{2}$ na estrutura do GO, o material comporta-se como um isolante elétrico. Contudo, a redução do GO a óxido de grafeno reduzido, rGO - condutivo, é obtida com a remoção dos grupos funcionais oxigenados e com o restabelecimento parcial de ligaçôes de carbono $\mathrm{sp}^{2}$. Para tanto, diferentes estratégias têm sido utilizadas na redução do GO, incluindo os processos químicos, térmicos, biológicos, fotoquímicos e eletroquímicos (AGARWAL; ZETTERLUND, 2021).

Uma das aplicaçôes promissoras do óxido de grafeno reduzido é a detecção eletroquímica, pois cada folha de grafeno transporta elétrons, que são altamente sensíveis às moléculas adsorvidas. Acredita-se que o grafeno, quando utilizado para a modificação dos eletrodos convencionais, tem grande potencial para distinguir os isômeros aromáticos (CHEN et al., 2011; SHAMKHALICHENAR; CHOI, 2020). 
Desse modo, o presente trabalho avaliou o comportamento eletroquímico da quercetina e da naringenina em diferentes valores $\mathrm{pH}$ sobre eletrodos de carbono vítreo e carbono vítreo modificado com rGO. Adicionalmente, as potencialidades do eletrodo modificado com rGO foram exploradas na identificação e avaliação da concentraçáo de quercetina em extrato butanólico da planta Ludwigia repens.

\section{PARTE EXPERIMENTAL}

\subsection{Soluçóes e reagentes}

Todas as soluçóes aquosas foram preparadas com água deionizada (resistividade > 18,2 M $2 . \mathrm{cm}$ ) obtida com sistema de purificação Smart Park Millipore. Os flavonoides e GO utilizados neste estudo foram adquiridos da Sigma-Aldrich. Os demais reagentes e solventes de grau analítico foram utilizados sem purificação prévia.

\subsection{Preparaçáo das soluçóes tampáo Britton Robinson (BR)}

Em um baláo volumétrico de $250 \mathrm{~mL}$ foram adicionados $1,953 \mathrm{~g} \mathrm{de} \mathrm{KCl}(0,10 \mathrm{~mol}$ $\left.\mathrm{L}^{-1}\right), 0,620 \mathrm{~g}$ de ácido bórico $\left(0,040 \mathrm{~mol} \mathrm{~L}^{-1}\right), 0,57 \mathrm{~mL}$ de ácido glacial $\left(0,040 \mathrm{~mol} \mathrm{~L}^{-1}\right)$ e $0,53 \mathrm{~mL}$ de ácido orto-fosfórico $\left(0,040 \mathrm{~mol} \mathrm{~L}^{-1}\right)$. Completou-se o volume do balão volumétrico com água deionizada e foram ajustados os valores do $\mathrm{pH} 2,63 ; 4,33 ; 6,41$ e 8,67 com $\mathrm{NaOH} 0,20 \mathrm{~mol} \mathrm{~L}^{-1}$.

\subsection{Preparaçáo da soluçáo tampáo fosfato}

Em um balão volumétrico de $10 \mathrm{~mL}$ foi adicionado $0,092 \mathrm{~g}$ de $\mathrm{KH}_{2} \mathrm{PO}_{4}(0,067$ mol L $\left.\mathrm{L}^{-1}\right)$ e o volume foi completado com água. Em outro baláo volumétrico de 100 $\mathrm{mL}$, adicionou-se $0,951 \mathrm{~g}$ de $\mathrm{NaHPO}_{4}\left(0,067 \mathrm{~mol} \mathrm{~L}^{-1}\right)$ e completou-se o volume com água. Uma alíquota de $1,0 \mathrm{~mL}$ da solução $\mathrm{KH}_{2} \mathrm{PO}_{4}$ foi adicionada a um balão volumétrico de $100 \mathrm{~mL}$ e completou-se o volume com a solução de $\mathrm{NaHPO}_{4}$. O pH foi ajustado com ácido ortofosfórico até o valor de 6,59 . 


\subsection{Preparaçáo da soluçáo tampáo ácido acético/acetato de sódio $\mathrm{pH} 4,7$}

Inicialmente, adicionou-se $0,17 \mathrm{~mL}$ de ácido acético glacial em um balão de 100 $\mathrm{mL}$ para obter uma solução de concentração $1,0 \mathrm{~mol} \mathrm{~L}^{-1}$. Depois, $10 \mathrm{~mL}$ dessa solução foram colocados num baláo de $100 \mathrm{~mL}$ e completou-se o volume com água deionizada, resultando numa concentração de $0,10 \mathrm{~mol} \mathrm{~L}^{-1}$. Para ajustar o pH, adicionou-se acetato de sódio sólido até este alcançar o valor de 4,75.

\subsection{Preparaçáo da soluçáo de quercetina}

Para a obtenção dos voltamogramas cíclicos, adicionaram-se, na célula eletroquímica, alíquotas de $7,5 \mathrm{~mL}$ da solução de quercetina $\left(2,0 \times 10^{-4} \mathrm{~mol} \mathrm{~L}^{-1}\right)$ em DMSO e 7,5 mL da solução tampáo BR, nos valores de $\mathrm{pH}$ de 2,63; 4,33; 6,41 e 8,67, respectivamente.

Para o estudo da mistura de flavonoides, adicionou-se 0,067 g de quercetina em um baláo volumétrico de $100 \mathrm{~mL}\left(2,0 \times 10^{-3} \mathrm{~mol} \mathrm{~L}^{-1}\right)$ e completou-se o volume com DMSO. Os estudos voltamétricos foram feitos em numa cela eletroquímica contendo $1,5 \mathrm{~mL}$ da soluçáo dos flavonoides e $13,5 \mathrm{~mL}$ da solução tampão fosfato $\mathrm{pH} \mathrm{6,95.}$

\subsection{Preparaçáo da solução de naringenina}

Para as medidas de voltametria cíclica, adicionaram-se na cela eletroquímica 1,5 $\mathrm{mL}$ da solução de naringenina $\left(1,8 \times 10^{-3} \mathrm{~mol} \mathrm{~L}^{-1}\right)$ em DMSO e $13,5 \mathrm{~mL}$ da solução de fosfato $\mathrm{pH} 6,95$.

\subsection{Instrumentação}

As medidas de voltametria cíclica foram realizadas com potenciostato/galvanostato da Autolab, modelo PGSTAT 302N conectado a um microcomputador e gerenciado pelo software NOVA (versão 1.11). Utilizou-se uma célula eletroquímica com três eletrodos: eletrodo de trabalho (carbono vítreo limpo e modificado com rGO); eletrodo de referência $(\mathrm{Ag} / \mathrm{AgCl})$, combinado com um capilar de Luggin; e um eletrodo auxiliar (bastáo de carbono vítreo). Todas as soluçóes analisadas foram previamente desareadas com nitrogênio durante 10 minutos. As medidas potenciométricas de $\mathrm{pH}$ 
foram realizadas com potenciômetro Digimed modelo DM-22, com eletrodo de vidro combinado e eletrodo de referência de $\mathrm{Ag} / \mathrm{AgCl}\left(\mathrm{KCl} 3,0 \mathrm{~mol} \mathrm{~L}^{-1}\right)$.

A caracterização do eletrodo carbono vítreo limpo e modificado com rGO foi feita através da técnica de espectroscopia Raman, utilizando espectrômetro e microscópio Witec, modelo Alpha 300R, com laser de $532 \mathrm{~nm}$ e as lentes de amplificação de $10 \mathrm{x}$ e $50 \mathrm{x}$.

\subsection{Modificaçáo eletroquímica do eletrodo de carbono vítreo com óxido de grafeno (GO)}

Sobre a superfície do eletrodo de carbono vítreo limpo (área $=2,0 \mathrm{~mm}^{2}$ ), adicionou-se uma gota $(60 \mu \mathrm{L})$ da solução do óxido de grafeno comercial $\left(2,0 \mathrm{mg} \mathrm{mL}^{-1}\right)$ e, então, submeteu-se o eletrodo à ação de uma lâmpada incandescente com potência de $100 \mathrm{~W}$ por um período de uma hora para a evaporação da solução e a formação do filme sobre a superfície do eletrodo. A redução eletroquímica do GO depositado sobre o eletrodo de carbono vítreo foi realizada em uma cela eletroquímica contendo $20 \mathrm{~mL}$ de solução tampão de ácido acético/acetato de sódio $0,10 \mathrm{~mol} \mathrm{~L}^{-1}, \mathrm{pH}=4,75$ desaerada com nitrogênio, na faixa de potencial de 0,0 a $-1,2 \mathrm{~V} v$ s. $\mathrm{Ag} / \mathrm{AgCl}$.

\section{RESULTADO E DISCUSSÃO}

\subsection{Medidas eletroquímicas dos flavonoides}

$\mathrm{O}$ voltamograma cíclico da quercetina (Figura 2) em solução tampão $\mathrm{BR} \mathrm{pH}=$ 6,41 usando um eletrodo de carbono vítreo apresenta três picos de oxidação em $0,24 \mathrm{~V}, 0,64 \mathrm{~V}$ e $0,89 \mathrm{~V}$ vs $\mathrm{Ag} / \mathrm{AgCl}$. O primeiro pico, em $0,24 \mathrm{~V}\left(\mathrm{E}_{\mathrm{p}}{ }_{\mathrm{p}}^{\mathrm{I}}\right)$, é quase reversível, conforme descrito por Sokolová et al. (2012). Os picos $\mathrm{E}_{\mathrm{p}}{ }_{\mathrm{II}}$ e $\mathrm{E}_{\mathrm{p}}^{\mathrm{III}}$ envolvem a oxidação do grupo hidroxila em C-3 e os substituintes 5,7-di-hidroxi no anel A (TIMBOLA et al., 2006), que é mais evidente com o aumento da velocidade de varredura (Figura 2-II). 

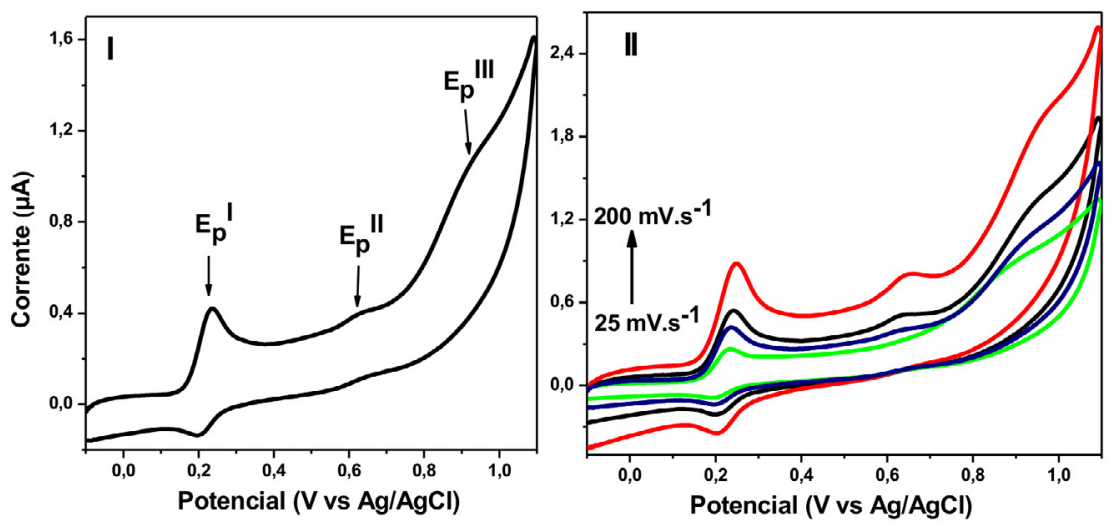

Figura 2 Voltamogramas cíclicos da quercetina $\left(1,0 \times 10^{-4} \mathrm{~mol} \mathrm{~L}^{-1}\right)$ em solução contendo tampão BR pH 6,4 em DMSO na proporção de 1:1, na região de 0 até 1,1 V, usando (I) velocidade de varredura de $50 \mathrm{mV} \mathrm{s}^{-1}$; (II) em diferentes velocidades de varreduras.

Fonte: Elaborada pelos autores.

$\mathrm{Na}$ Figura 2-I, são mostrados os voltamogramas cíclicos da quercetina $\left(\mathrm{QH}_{2}\right)$ em soluçóes tampão BR com valores de $\mathrm{pH} 2,63 ; 4,33 ; 6,41$ e 8,67, nos quais se observam até três picos de oxidaçáo, que se deslocam para potenciais menores com o aumento no valor de $\mathrm{pH}$ (Tabela 1 ).

\section{TABELA 1}

Valores de potencial do primeiro pico de oxidação $\left(E_{p a}{ }^{\prime}\right)$, do segundo $\left(E_{p a}{ }^{\prime \prime}\right)$ e do terceiro

$\left(E_{p a}{ }^{\text {III }}\right)$ da quercetina em função dos valores de $\mathrm{pH}$, obtidos por voltametria cíclica, a partir de soluções da quercetina em DMSO e tampão BR na proporção de 1:1, em 50 mV.s.-1.

$\begin{array}{cccc}p H & E_{p a}^{\prime}(V) & E_{p a}^{\prime \prime}(V) & E_{p a}^{\prime \prime \prime}(V) \\ 2,63 & 0,46 & 0,74 & - \\ 4,33 & 0,36 & 0,75 & - \\ 6,41 & 0,24 & 0,62 & 0,95 \\ 8,67 & 0,08 & 0,59 & 0,87\end{array}$

O deslocamento dos potenciais dos picos de oxidação para valores menos positivos com o aumento do $\mathrm{pH}$ sugere, ainda, um aumento da nucleofilicidade da espécie eletroativa e que a atividade antioxidante da quercetina é termodinamicamente favorecida com o acréscimo do pH (BRETT; BRETT, 1993). 

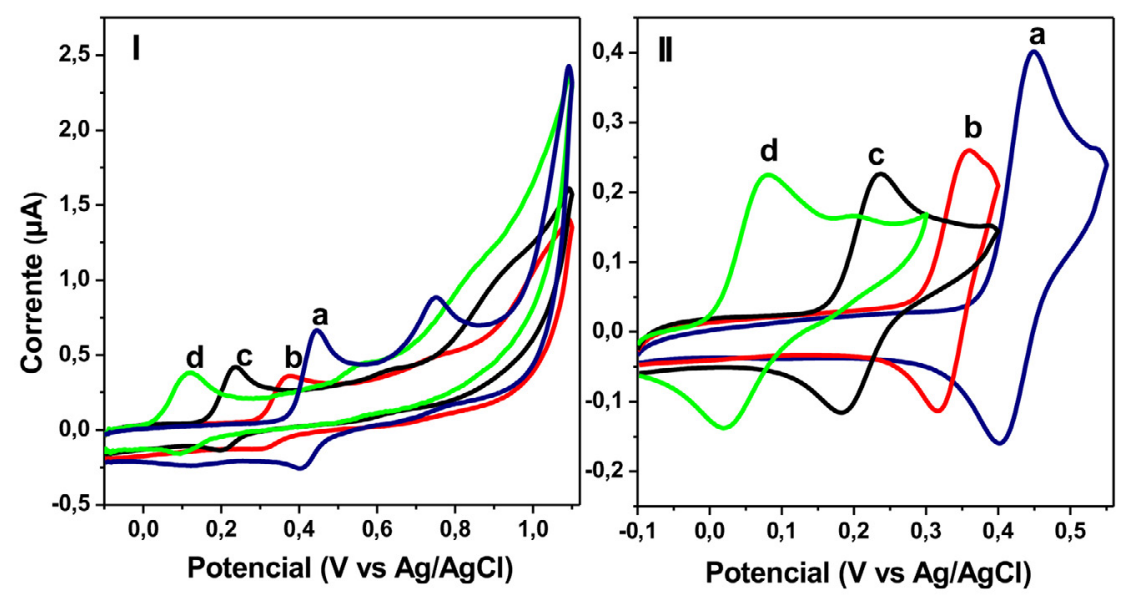

Figura 3 Voltamogramas cíclicos da quercetina $\left(1,0 \times 10^{-4} \mathrm{~mol} \mathrm{~L}^{-1}\right)$ em solução contendo tampão BR em diferentes pHs (a) 2,63 (b) 4,33 (c) 6,41 e (d) 8,67 e DMSO na proporção de 1:1, na região de 0,10 até $1,1 \mathrm{~V}$ (I) e -0,10 até $0,56 \mathrm{~V}$ (II), usando velocidade de varredura de $50 \mathrm{mV} \mathrm{s}^{-1}$.

Fonte: Elaborada pelos autores.

$\mathrm{Na}$ Tabela 2, encontram-se os valores de potencial de pico anódico $\left(\mathrm{E}_{\mathrm{pa}}\right)$, o potencial de meia onda $\left(\mathrm{E}_{1 / 2}\right)$ e a relação das correntes de picos anódico e catódico. Os processos voltamétricos, particularmente aqueles associados a sistemas orgânicos, em alguns casos são parciais ou totalmente irreversíveis, o que produz curvas mal definidas.

$\mathrm{O}$ voltamograma da Figura 3-II representa um sistema quase reversível com o primeiro pico de oxidaçáo em $0,46 \mathrm{~V}(\mathrm{pH} 2,63)$ e um pico de redução em $0,41 \mathrm{~V}$. Esse pico anódico é atribuído, de acordo com a literatura (TIMBOLA et al., 2006), à oxidação dos grupos 3', '4'-di-hidroxi do anel B (Figura 1-A).

Os valores de potencial desse primeiro pico de oxidação $\left(\mathrm{E}_{\mathrm{pa}}{ }^{\mathrm{I}}\right)$ apresentam uma dependência linear com o pH (Figura 4), cujo coeficiente linear, igual a $0,62 \mathrm{~V} v$ s. $\mathrm{Ag} / \mathrm{AgCl}$, é relativo ao potencial redox padrão desse primeiro processo de oxidação (Equação 1). O coeficiente angular tem um valor de -0,0617 V. Esse valor é próximo ao esperado pela equaçáo de $\operatorname{Nernst~}(-0,0592 \mathrm{~V})$, indicando que o mesmo número de prótons e elétrons está envolvido no processo de oxidação, o qual pode ser descrito pela Equação 2. 


$$
\begin{gathered}
\mathrm{E}_{\mathrm{pa}}^{\mathrm{I}}(\mathrm{V})=(0,62 \pm 0,01)-(0,0617 \pm 0,002) \mathrm{pH}(\mathrm{R}=0,998) \\
\mathrm{QH}_{2} \rightarrow \mathrm{Q}+2 \mathrm{H}^{+}+2 \mathrm{e}^{-}
\end{gathered}
$$

\section{TABELA 2}

Valores de potencial do primeiro pico de oxidação $\left(E_{p a}{ }^{\prime}\right)$ da quercetina em função dos valores de $\mathrm{pH}^{*}$, potencial de meia-onda $\left(\mathrm{E}_{1 / 2}\right)$ e a relação entre as correntes de pico $\left(\mathrm{i}_{\mathrm{pa}} / \mathrm{i}_{\mathrm{pc}}\right.$, obtida por voltametria cíclica a partir de soluções da quercetina em DMSO e tampão BR na proporção

\begin{tabular}{|c|c|c|c|c|c|c|}
\hline \multicolumn{4}{|c|}{$\mathrm{E}_{\mathrm{pa}}^{\prime}(\mathrm{V})$} & \multicolumn{2}{|c|}{$E_{1 / 2}(V)$} & \multirow{2}{*}{$\begin{array}{l}\quad \mathrm{i}_{\mathrm{pa}} / \mathrm{i}_{\mathrm{pc}}(\mathrm{A}) \\
\text { Este trabalho }\end{array}$} \\
\hline Este trabalho & Ref $1 * *$ & Ref. $2 * *$ & Ref $3 * *$ & Este trabalho & Ref. 3** & \\
\hline $0,46(2,63)$ & - & $0,48(2,2)$ & $0,39(3,5)$ & 0,43 & $0,34(3,5)$ & 0,93 \\
\hline $0,36(4,33)$ & $0,38(4,3)$ & $0,35(4,4)$ & $0,32(4,5)$ & 0,33 & $0,27(4,5)$ & 0,46 \\
\hline $0,24(6,41)$ & $0,23(6,1)$ & $0,23(6,5)$ & $0,25(5,5)$ & 0,21 & $0,19(5,5)$ & 1,07 \\
\hline $0,08(8,67)$ & $0,16(8,9)$ & $0,10(8,4)$ & $0,07(8,0)$ & 0,05 & $0,05(8,0)$ & 1,50 \\
\hline
\end{tabular}
de $1: 1$ em $50 \mathrm{mV} \mathrm{s}^{-1}$.

* Valores de $\mathrm{pH}$ entre parênteses.

** $25 \mathrm{mV} . \mathrm{s}^{-1}$ (SOKOLOVÁ, R. et al., 2012); $100 \mathrm{mV} . \mathrm{s}^{-1}$ (TIMBOLA et al., 2006); $100 \mathrm{mV} . \mathrm{s}^{-1}$ (KOSANOVIĆ, M. M. et al.).

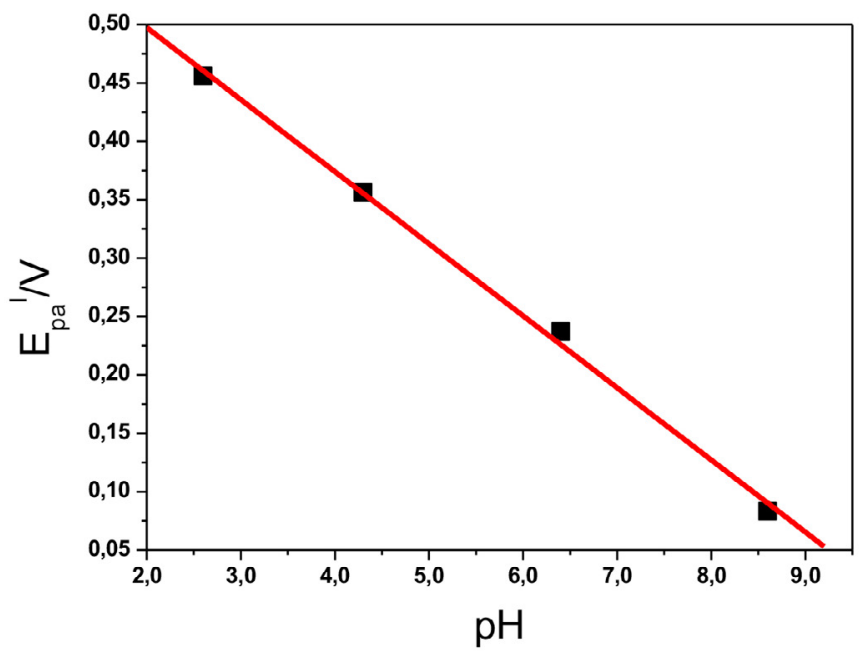

Figura 4 Perfil da variação do potencial de pico anódico em função do pH, obtido a partir de uma solução $1,0 \times 10^{-4} \mathrm{~mol} \mathrm{~L}^{-1}$ de quercetina contendo DMSO e tampão BR, na proporção de 1:1, usando velocidade de varredura de $50 \mathrm{mV} \mathrm{s}^{-1}$. 
Sistemas reversíveis ou quase reversíveis, em que a transferência de elétrons é seguida de desprotonação (LOURENÇO, 2004), seguem a Equação 3, que relaciona a etapa de oxidação $(\mathrm{Ep})$, o potencial redox $\left(\mathrm{E}^{\circ}\right)$, o número de elétrons (n) e o número de prótons (a) ao $\mathrm{pH}$.

$$
\mathrm{Ep}=\mathrm{E}^{\mathrm{o}}-(\mathrm{a} / \mathrm{n}) 0,0592 \mathrm{pH}
$$

O voltamograma cíclico da naringenina, em solução hidroalcoólica contendo tampão $\mathrm{BR}$ pH = 5,45 (Figura 5-I), obtido com eletrodo de carbono vítreo, mostra um pico de oxidação irreversível em $0,82 \mathrm{~V}$. $\left(\mathrm{E}_{\mathrm{p}}^{\mathrm{I}}\right)$, que, de acordo com a literatura (CHENG; BREEN, 2000), é atribuida à oxidaçáo do grupo 4'-hidroxi no anel B (Figura 1-B).

Observou-se um deslocamento do potencial do pico de oxidação (Figura 5-II) da naringenina para potenciais menores com o aumento do $\mathrm{pH}$ (Tabela 3), indicando que a oxidaçáo envolve um processo de desprotonaçáo que depende do $\mathrm{pH}$ do meio (SMITH, 2006).
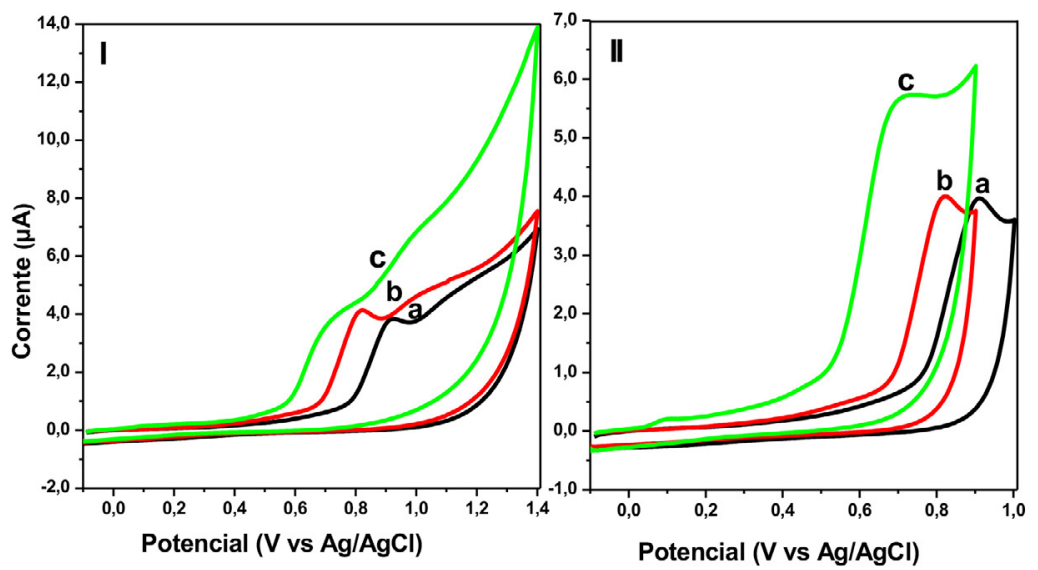

Figura 5 Voltamogramas cíclicos da naringenina $\left(1,0 \times 10^{-3} \mathrm{~mol} \mathrm{~L}^{-1}\right)$ em solução contendo tampão BR em diferentes pHs (a) 4,13 (b) 5,45 e (c) 8,01 DMSO na proporção de 1:1, na região de 0,10 até $1,40 \mathrm{~V}$ (I) e -0,10 até 1,0 V (II), usando velocidade de varredura de $25 \mathrm{mV} \mathrm{s}^{-1}$.

Fonte: Elaborada pelos autores.

A Figura 6 mostra a dependência linear do potencial do pico de oxidação da naringenina com o $\mathrm{pH}$, cuja equação da reta obtida por regressão linear é representada pela Equação 4: 


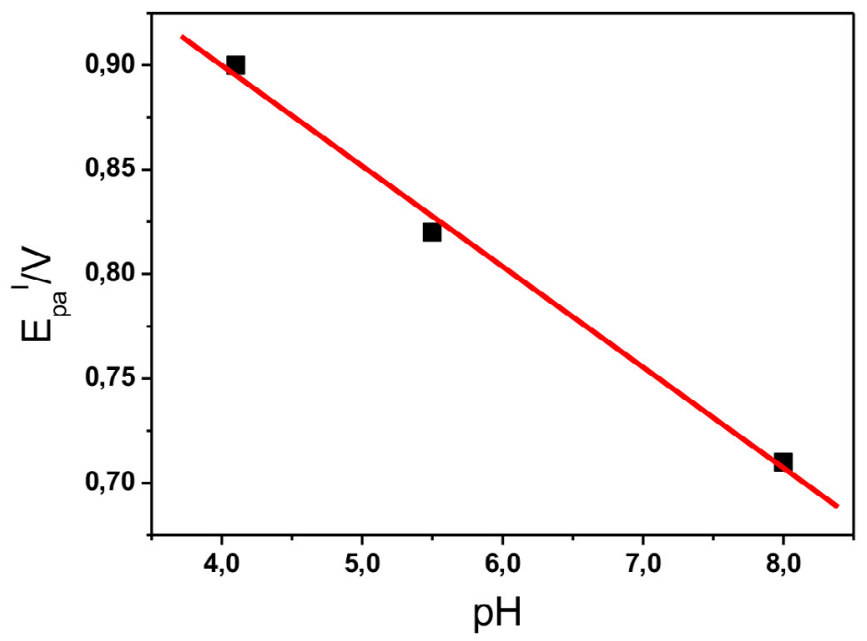

Figura 6 Perfil da variação do pico anódico em função do pH, obtido a partir de uma solução $1,0 \times 10^{-3} \mathrm{~mol} \mathrm{~L}^{-1}$ naringenina contendo DMSO e tampão BR, na proporção de $1: 1$, usando velocidade de varredura de $25 \mathrm{mV} \mathrm{s}^{-1}$.

$$
\mathrm{E}_{\mathrm{p}}(\mathrm{V})=(1,09 \pm 0,02)-(0,048 \pm 0,003) \cdot \mathrm{pH}(\mathrm{R}=0,997)
$$

\section{TABELA 3}

Valores de potencial do primeiro pico de oxidação $\left(\mathrm{E}_{\mathrm{pa}}{ }^{\prime}\right)$ e das correntes de pico $\left(\mathrm{i}_{\mathrm{pa}}\right)$ obtidos por voltametria cíclica a partir de soluções da naringenina em DMSO e tampão BR na proporção de 1:1 em $25 \mathrm{mV} . \mathrm{s}^{-1}$.

\begin{tabular}{ccccc}
$\mathrm{pH}$ & $\mathrm{E}_{\mathrm{pa}}{ }^{\prime}(\mathbf{V})$ & $\mathrm{I}_{\mathrm{pa}}(\mathbf{A})$ & $\left|\mathrm{E}_{\mathrm{p}}-\mathrm{E}_{\mathrm{p} / 2}\right|(\mathbf{V})$ & an \\
\hline 4,13 & 0,90 & $3,97 \times 10^{-6}$ & 45 & 0,94 \\
5,45 & 0,82 & $4,01 \times 10^{-6}$ & 41 & 0,85 \\
8,01 & 0,71 & $5,73 \times 10^{-6}$ & 35 & 0,73 \\
\hline
\end{tabular}

É possível calcular o valor do coeficiente de transferência de carga (an) para o intervalo de $\mathrm{pH}$ de 4,13 a 8,01 por meio da Equação 5 (Tabela 3) válida para sistemas irreversíveis (CAVALCANTE et al., 2005; BRETT; BRETT, 1993). 


$$
|\mathrm{Ep}-\mathrm{Ep} / 2|=\frac{47,7}{\mathrm{an}_{\mathrm{n}}}
$$

O número de prótons envolvidos na reação de oxidação do naringenina pode ser calculado pela expressão (Equação 6):

$$
\frac{\Delta \mathrm{E}_{\mathrm{p}}}{\Delta \mathrm{pH}}=-\frac{59,16 \mathrm{H}^{+}}{\mathrm{an}_{\mathrm{n}}}
$$

Utilizando o valor de an de $0,73(\mathrm{pH} 8,01)$, calculado por meio da inclinação da reta Ep vs. pH (Equação 5), o número de prótons $\mathrm{H}^{+}$é $1,11\left(1 \mathrm{H}^{+}\right)$. Segundo Mülazımoğlu, Özkan e Solak (2011), o processo apresenta o mesmo número de elétrons e prótons, assim, o mecanismo de reação de oxidação correspondente da naringenina $(\mathrm{RH})$ envolve a transferência de 1 próton $\mathrm{H}^{+}$e $1 \mathrm{e}^{-}$(Equação 7$)$.

$$
\mathrm{RH} \rightarrow \mathrm{R}+\mathrm{H}^{+}+\mathrm{e}^{-}
$$

\subsection{Redução eletroquímica do óxido de grafeno sobre o eletrodo de carbono vítreo}

Os voltamogramas cíclicos, obtidos durante a redução do GO sobre eletrodo de carbono vítreo, apresentaram um incremento na intensidade do pico catódico conforme aumentou o número de varreduras e estabilizou-se em aproximadamente - $0,70 \mathrm{~V}$ vs. $\mathrm{Ag} / \mathrm{AgCl}$ (Figura 7). O fato de o sinal de corrente de pico catódico aumentar após varreduras sucessivas é um indício da deposição de GO reduzido sobre o eletrodo (CHEN et al., 2011). 


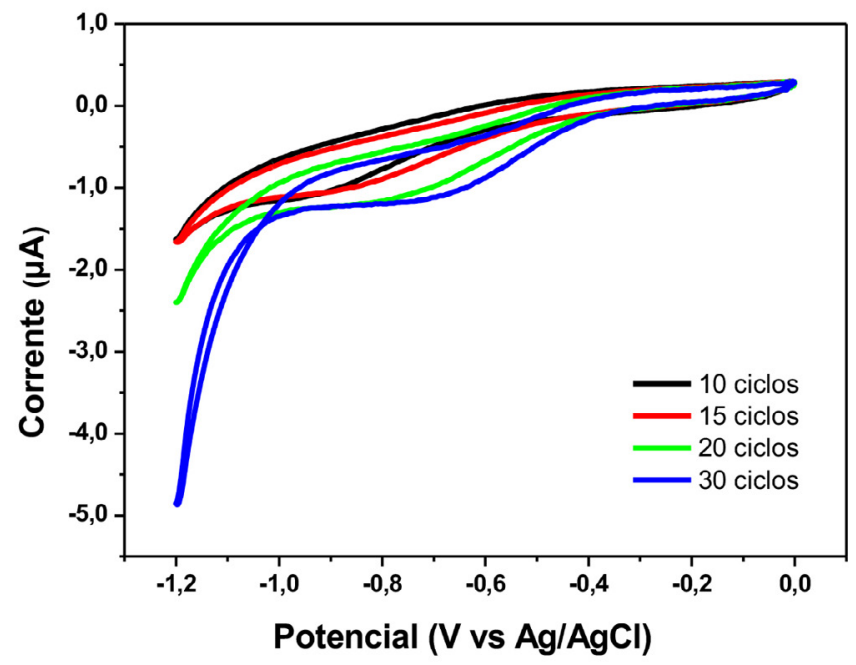

Figura 7 Voltamogramas cíclicos mostrando a redução eletroquímica do GO depositado sobre o eletrodo de carbono vítreo em solução tampão acetato, pH 4,75, após 10, 15, 20 e 30 varreduras a $10 \mathrm{mV} \cdot \mathrm{s}^{-1}$.

Fonte: Elaborada pelos autores.

O voltamograma cíclico da solução $10 \mathrm{mmol} \mathrm{L}{ }^{-1}$ de $\left[\mathrm{K}_{3}\left(\mathrm{FeCN}_{6}\right)\right]$ obtido usando o eletrodo de carbono vítreo modificado com rGO apresenta uma menor separaçáo entre os picos, $\Delta \mathrm{E}$, de $90 \mathrm{mV}$ vs. $\mathrm{Ag} / \mathrm{AgCl}$ para $\mathrm{rGO} / \mathrm{GCE}$ contra $110 \mathrm{mV}$ vs. $\mathrm{Ag} / \mathrm{AgCl}$ para GCE, e uma maior intensidade de corrente (Figura 8). O menor valor de $\Delta \mathrm{E}$ observado está relacionado ao aumento na cinética de transferência de elétrons, devido ao restabelecimento das ligaçóes $\pi$ do grafeno, após o processo de redução dos grupos oxigenados, o que torna a superfície do eletrodo mais condutora (WANG et al., 2009). $\mathrm{O}$ aumento na intensidade dos sinais de corrente de pico anódica $\mathrm{e}$ catódica para o par redox $\left[\mathrm{Fe}(\mathrm{CN})_{6}\right]^{3-} /\left[\mathrm{Fe}(\mathrm{CN})_{6}\right]^{4-}$ sugere o aumento de sítios eletroativos na superfície do eletrodo modificado, decorrentes de defeitos no filme de rGO resultante dos grupamentos de oxigênio restantes nas estrutura de grafeno (CHEN et al., 2011). 


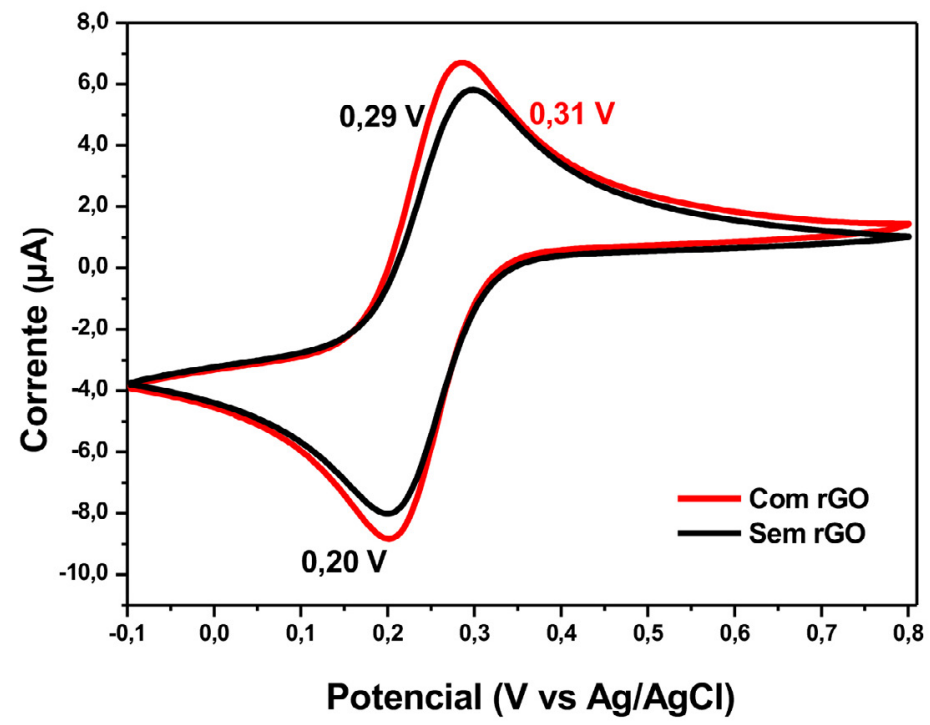

Figura 8 Voltamogramas cíclicos de solução $10 \mathrm{mmol} \mathrm{L}^{-1}$ de $\left[\mathrm{K}_{3}\left(\mathrm{FeCN}_{6}\right)\right]$ em $\mathrm{KCl} 0,10 \mathrm{~mol} \mathrm{~L}^{-1}$, usando eletrodo de carbono vítreo sem e com rGO a $25 \mathrm{mVs}^{-1}$.

Fonte: Elaborada pelos autores.

Os resultados da espectroscopia Raman (Figura 9) mostram que o eletrodo de carbono vítreo não modificado apresenta bandas em $1345 \mathrm{~cm}^{-1}(\mathrm{D}), 1577 \mathrm{~cm}^{-1}(\mathrm{G})$, $2665 \mathrm{~cm}^{-1}\left(\mathrm{G}^{\prime}\right), 2915 \mathrm{~cm}^{-1}$ (D+G) e $3214 \mathrm{~cm}^{-1}$ (2D'). Após redução eletroquímica do GO depositado sobre o eletrodo GCE, observa-se uma mudança nas bandas G' e $\mathrm{D}+\mathrm{G}$ que, respectivamente, diminuem e aumentam de intensidade. Observa-se também um alargamento das bandas G', que indica maior reestruturação da estrutura grafítica nesse material reduzido. $\mathrm{O}$ mesmo aconteceu com a banda $\mathrm{D}+\mathrm{G}$, sugerindo maior heterogeneidade ou desordem da estrutura (DOMINGUES, 2013; TOH et al., 2014). 


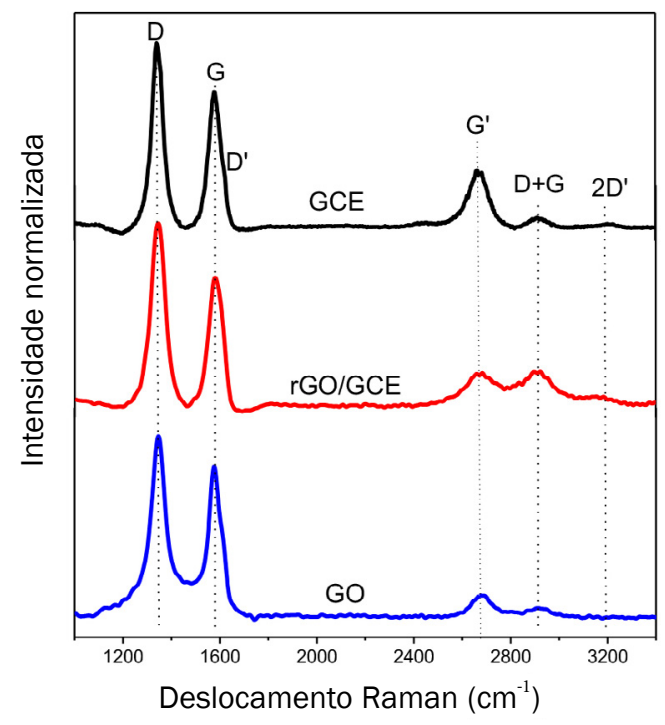

Figura 9 Espectros Raman do eletrodo de carbono vítreo (GCE); eletrodo modificado (rGO/GCE) e do óxido de grafeno (GO) sobre substrato de $\mathrm{SiO}_{2}$ usando laser de $532 \mathrm{~nm}$.

Fonte: Elaborada pelos autores.

A Figura 10-I ilustra os voltamogramas cíclicos da quercetina em solução tampão fosfato, $\mathrm{pH} 6,95$, obtidos no intervalo de potencial de $-0,10 \mathrm{~V}$ a $1,40 \mathrm{~V}$ com eletrodo de carbono vítreo não modificado e modificado com rGO. Observam-se dois picos anódicos em $0,17 \mathrm{~V}$ e $-0,92 \mathrm{~V}$ e um pico catódico discreto em $0,13 \mathrm{~V}$, evidenciado na Figura 10-IA. O perfil dos sinais de corrente não se altera com o uso dos dois materiais de eletrodo. No entanto, a intensidade da corrente anódica aumenta com o eletrodo de rGO, indicando que a oxidação da quercetina é sensível à modificação do eletrodo. Quando se restringe a faixa de potencial ao intervalo de $-0,10$ a $-0,30 \mathrm{~V}$, o pico catódico em $0,13 \mathrm{~V}$ torna-se mais bem definido e a intensidade dos sinais de pico anódico e catódico aumenta com uso do eletrodo rGO/GCE, sugerindo que os grupos oxigenados no eletrodo melhoram a interação com a quercetina na interface eletrodo-solução. 

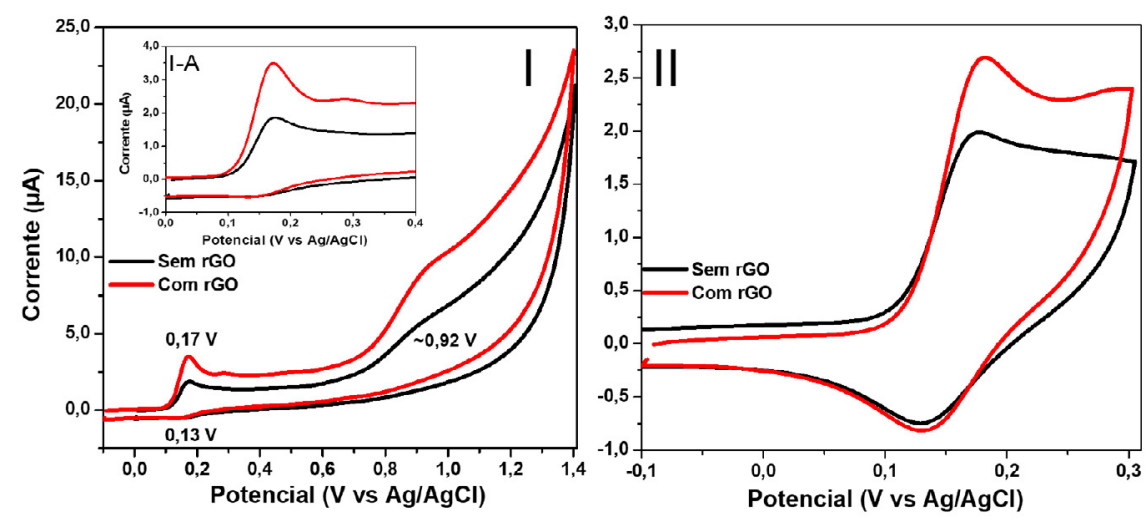

Figura 10 Voltamogramas cíclicos da quercetina $\left(2,0 \times 10^{-4} \mathrm{~mol} \mathrm{~L}^{-1}\right)$ em solução contendo DMSO e tampão fosfato pH 6,95 na proporção de 1:9 (a) sem o rGO e (b). Eletrodo rGO/GCE, usando velocidade de varredura de $50 \mathrm{mV} \mathrm{s}^{-1}$ e faixa de potencial de $-0,10$ a $1,40 \mathrm{~V}$ (I) e - 0,10 a $0,30 \mathrm{~V}(\mathrm{II})$.

Fonte: Elaborada pelos autores.

O voltamograma cíclico da naringenina (Figura 11) apresenta dois picos anódicos mal definidos em $0,76 \mathrm{~V}$ e $0,97 \mathrm{~V}$ vs. $\mathrm{Ag} / \mathrm{AgCl}$, próximos aos valores encontrados na literatura (ZHANG et al., 2011), respectivamente iguais a $0,710 \mathrm{~V}$ e $0,955 \mathrm{~V}$ vs $\mathrm{Ag} / \mathrm{AgCl}$. Assim como aconteceu com a quercetina, as medidas realizadas com o eletrodo modificado apresentaram maior intensidade de corrente. Quando se restringe a faixa de potencial ao intervalo de $-0,10$ a $0,80 \mathrm{~V}$ (Figura 11-II), um discreto sinal anódico e um catódico é observado com mais clareza em $\sim 0,22 \mathrm{~V}$ quando se utiliza o eletrodo de rGO, sugerindo a presença de um par redox eletroativo de um grupamento de oxigênio estável na superfície de grafeno que não foi reduzido durante a etapa eletroquímica de formação do filme de rGO (HUMMERS; OFFEMAN, 1958; MARRANI et al., 2019). 

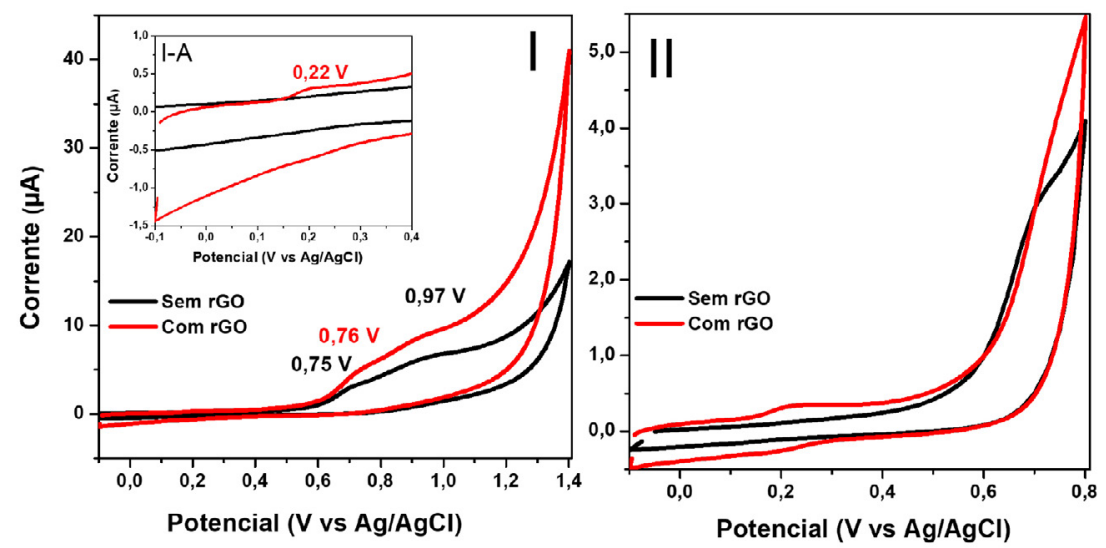

Figura 11 Voltamogramas cíclicos da naringenina $\left(1,8 \times 10^{-4} \mathrm{~mol} \mathrm{~L}^{-1}\right)$ em solução contendo DMSO e tampão fosfato $\mathrm{pH} 6,95$ na proporção de 1:9 usando velocidade de varredura de $50 \mathrm{mV} \mathrm{s}^{-1} \mathrm{e}$ faixa de potencial de $-0,10$ a $1,40 \mathrm{~V}$ (I) e $-0,10$ a $0,80 \mathrm{~V}$ (II).

Fonte: Elaborada pelos autores.

\subsection{Identificaçáo de quercetina e narigenina utilizando o eletrodo de rGO/GCE}

Com o intuito de avaliar a viabilidade da utilização do eletrodo de rGO/GCE para identificar a presença de flavonoides, como a quercetina e a naringenina em extratos vegetais, utilizou-se um extrato aquoso da espécie vegetal Ludwigia repens, que contém compostos de elevada polaridade e ocorrência de flavonoides.

$\mathrm{Na}$ comparaçáo do sinal do extrato vegetal com o pico da quercetina, nota-se que o pico extrato vegetal ocorre em potenciais mais positivos que o da quercetina, $0,17 \mathrm{~V}$ (Figura 12), enquanto não é possível observar o pico da naringenina situado entre $0,76 \mathrm{~V}$ e $0,97 \mathrm{~V}$ vs. $\mathrm{Ag} / \mathrm{AgCl}$. Como o extrato tem um pico anódico bem definido em $0,35 \mathrm{~V}$, fez-se a adição de quercetina solubilizada em DMSO na solução contendo o extrato para avaliar a presença do flavonoide na amostra. A Figura 13-I mostra os voltamogramas cíclicos obtidos com a amostra do extrato vegetal e a adição de quantidades crescentes quercetina no intervalo de concentração de 118 a $471 \mu \mathrm{mol} \mathrm{L}{ }^{-1}$. Observa-se um aumento do sinal de corrente anódica a partir de 0,090 V e que se intensifica com a adição de quercetina ao meio. 


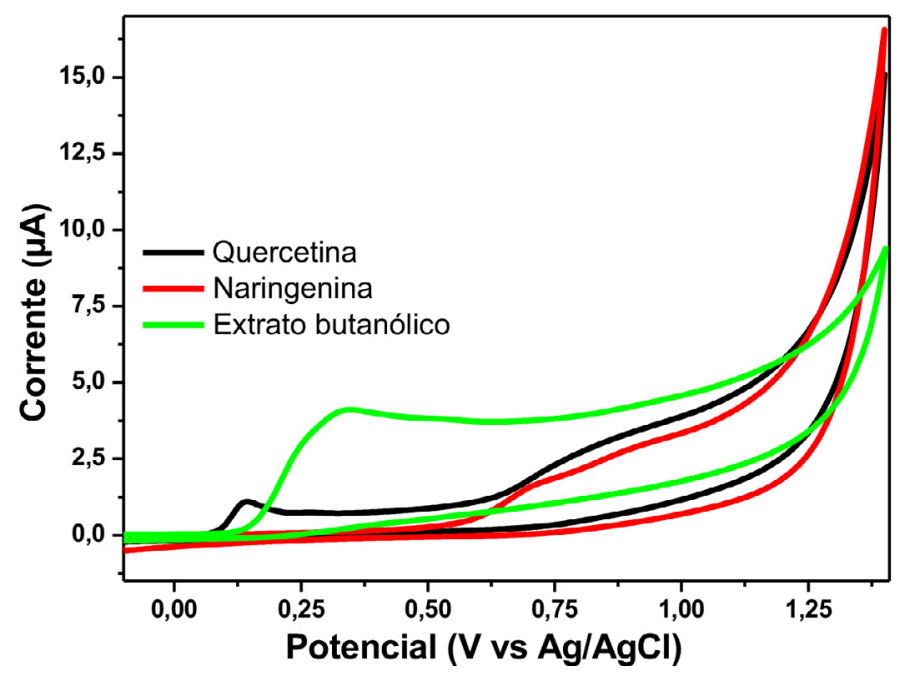

Figura 12 Voltamogramas cíclicos em solução contendo DMSO e tampão fosfato pH 6,95 na proporção de $1: 9$ da naringenina $\left(1,8 \times 10^{-4} \mathrm{~mol} \mathrm{~L}^{-1}\right)$, quercetina $\left(2,0 \times 10^{-4} \mathrm{~mol} \mathrm{~L}^{-1}\right)$, extrato butanólico (30 $\mathrm{mg} \mathrm{mL}^{-1}$ ), com eletrodo modificado com rGO, usando velocidade de varredura de $25 \mathrm{mV} \mathrm{s}^{-1}$ e faixa de potencial de $-0,10$ a $1,40 \mathrm{~V}$.

Fonte: Elaborada pelos autores.

O gráfico do sinal de corrente anódica medida em $0,30 \mathrm{~V} v$ s. $\mathrm{Ag} / \mathrm{AgCl}$ (Figura 13), em função da concentração da quercetina adicionada ao extrato butanólico, obedece a equação de reta:

$$
\mathrm{I}_{\mathrm{pa}}(\mu \mathrm{A})=(2,36 \pm 0,04)+(0,0028 \pm 0,0001) \cdot[\mathrm{Q}], \mu \mathrm{mol} \mathrm{L}^{-1}(\mathrm{R}=0,996)
$$

A extrapolação da reta média ao eixo $\mathrm{X}$ permitiu estimar a concentração de quercetina em $842,8 \mu \mathrm{mol} \mathrm{L}^{-1}$ (Figura 13-II). A análise dos resultados indica uma concentração significativa de quercetina na amostra avaliada. O extrato butanólico da planta Ludwigia Repens será estudado por outras técnicas analíticas visando avaliar de forma mais adequada as espécies presentes. 

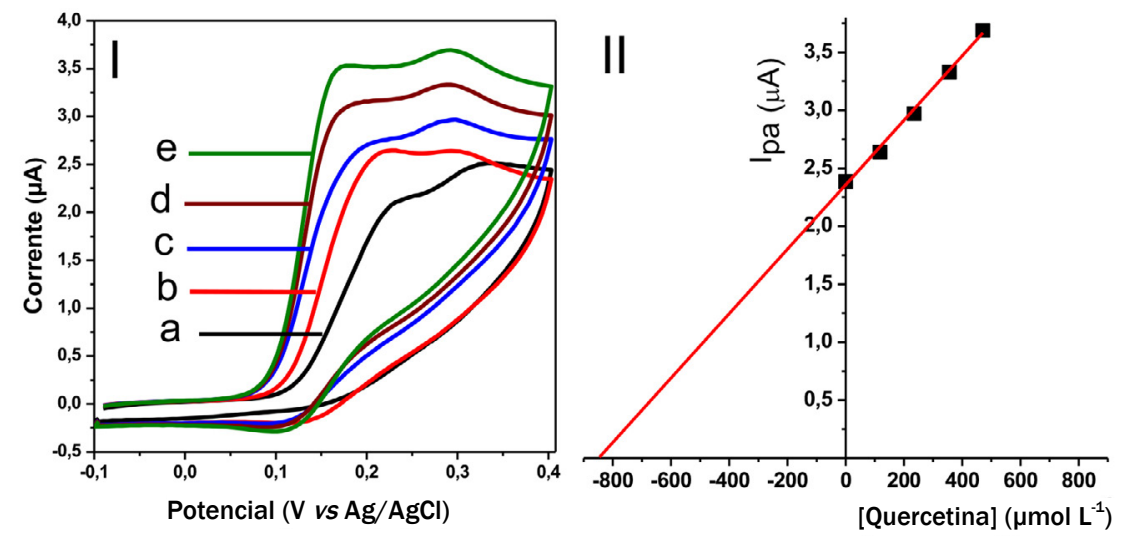

Figura 13 Voltamogramas cíclicos obtido com extrato butanólico (10 mg mL $\mathrm{mL}^{-1}$ ) em solução de DMSO e tampão fosfato pH 6,95 na proporção de 1:9 (a) e com adições de concentração crescente de quercetina na amostra: (b) $118 \mu \mathrm{mol} \mathrm{L}^{-1}$; (c) $235 \mu \mathrm{mol} \mathrm{L} \mathrm{L}^{-1}$; (d) $356 \mu \mathrm{mol} \mathrm{L}{ }^{-1}$; (e) 471 $\mu \mathrm{mol} \mathrm{L} \mathrm{L}^{-1}$. Velocidade de varredura de $25 \mathrm{mV} \mathrm{s}^{-1}$. (II) Determinação da concentração de quercetina no extrato vegetal pelo método de adição de padrão.

\section{CONCLUSÃO}

Este trabalho avaliou o comportamento eletroquímico dos flavonoides quercetina e naringenina em soluçóes com diferentes concentraçóes hidrogeniônicas. Os resultados mostraram que a quercetina tem três picos de oxidação. $\mathrm{O}$ potencial do primeiro pico de oxidação $\left(\mathrm{E}_{\mathrm{pa}}{ }^{\mathrm{I}}\right)$ apresenta uma dependência linear com o $\mathrm{pH}$, conforme a equação de Nernst, que indica que o mesmo número de prótons e elétrons está envolvido no processo de oxidação da quercetina. A voltametria da naringenina indica um pico de oxidação, envolvendo a transferência de 1 próton $\mathrm{H}^{+}$e $1 \mathrm{e}^{-}$.

O processo eletroquímico de modificação dos eletrodos é simples, rápido, ambientalmente adequado, opera a temperatura ambiente e não demanda etapas de purificação do filme de rGO resultante. A resposta voltamétrica da quercetina e naringenina obtida com eletrodos modificados com rGO mostrou maior intensidade nos sinais de corrente.

O eletrodo modificado apresentou bom desempenho analítico na determinação de quercetina em extrato vegetal. 


\title{
ELECTROCHEMICAL STUDY OF THE QUERCETIN AND NARINGENIN FLAVONOIDS
}

\begin{abstract}
This article evaluates the electrochemical behavior of quercetin and naringenin flavonoids in solutions with different $\mathrm{pH}$ values. A glassy carbon electrode (GCE) and a reduced graphene oxide-modified glassy carbon electrode (rGO/GCE) were used as the working electrodes. In a Britton-Robinson buffer solution, with $\mathrm{pH}$ 6.41, quercetin shows three oxidation peaks on a bare glassy carbon electrode (GCE), increasing signals current with the scan rate. Cyclic voltammetry measurements with both flavonoids at rGO/GCE electrode showed higher current intensity than those carried with GCE. Assays voltammetric carried out with (rGO/GCE) showed that between $0,0 \mathrm{~V}$ and $0,30 \mathrm{~V}$ vs. $\mathrm{Ag} / \mathrm{AgCl}$, the naringin does not interfere at the quercetin electrochemical detection in the vegetal extract of Ludwigia repens.
\end{abstract}

Keywords: Naringenin. Modified electrode. Quercetin. Reduced graphene oxide.

\section{REFERÊNCIAS}

AGARWAL, V.; ZETTERLUND, P. Strategies for reduction of graphene oxide: a comprehensive review. Chemical Engineering Journal, v. 405, n. 1, p. 127018, Feb. 2021. Disponível em: https:// www.sciencedirect.com/science/article/pii/S1385894720331466. Acesso em: 11 ago. 2021.

BRETT, C. M. A.; BRETT, A. M. O. Electrochemistry: Principles, Methods and Applications. New York: Oxford University Press, 1993.

CAVALCANTE, R. P. et al. Modificação química do eletrodo de pasta de carbono para detecção voltamétrica de metamidofós. 2005. Iniciação Científica (Graduação em Química) - Universidade Estadual de Mato Grosso do Sul, Dourados, 2005. Disponível em: https://anaisonline. uems.br/index.php/enic/article/view/1051. Acesso em: 11 ago. 2021.

CHAVES, H. M. et al. Fenóis totais e atividade antioxidante de cinco plantas medicinais. Quimica Nova, v. 30, n. 2, p. 351-355, abr. 2007. Disponível em: https://www.scielo.br/j/ qn/a/4d6yvCyDmhHyfP9ZJqV6nDy/?lang=pt. Acesso em: 11 ago. 2021.

CHEN, L. et al. Direct electrodeposition of reduced graphene oxide on glassy carbon electrode and its electrochemical application. Electrochemistry Communications, v. 13, n. 2, p. 133-137, Feb. 2011. Disponível em: https:/www.sciencedirect.com/science/article/pii/ S1388248110005072?via\%3Dihub. Acesso em: 11 ago. 2021. 
CHENG, F.; BREEN, K. On the ability of four flavonoids, baicilein, luteolin, naringenin, and quercetin, to suppress the Fenton reaction of the iron-ATP complex. Biometals, v. 13, p. 77-83, 2000. Disponível em: https://link.springer.com/content/pdf/10.1023/A:1009229429250.pdf. Acesso em: 11 ago. 2021.

CLASS FOR PHYSICS OF THE ROYAL SWEDISH ACADEMY OF SCIENCES (Comp.). Scientific Background on the Nobel Prize in Physics 2010: Graphene. Stockholm: The Royal Swedish Academy of Sciences, 2010. Disponível em: https://www.nobelprize.org/uploads/2018/06/advanced-physicsprize2010.pdf. Acesso em: 9 maio 2019.

DOMINGUES, S. H. Filmes finos, transparentes e condutores baseados em grafeno. 2013. Tese (Doutorado em Química) - Universidade Federal do Paraná, Curitiba, 2013. Disponível em: https://acervodigital.ufpr.br/bitstream/handle/1 884/36578/R\%20-\%20T\%20-\% 20SERGIO\%20HUMBERTO\%20DOMINGUES.pdf?sequence=1\&isAllowed=y. Acesso em: 11 ago. 2021.

GUAN, F. et al. Anti-rheumatic effect of quercetin and recent developments in nano formulation. Royal Society of Chemistry, v. 11, p. 7280-7293, Feb. 2021. Disponível em: https://pubs. rsc.org/en/content/articlelanding/2021/ra/d0ra08817j. Acesso em: 11 ago. 2021.

HUMMERS, S. J. W.; OFFEMAN, E. R. Preparation of Graphitic Oxide. Journal of the American Chemical Society, v. 80, n. 6, p. 1339, Mar. 1958. Disponível em: https://pubs.acs. org/doi/pdf/10.1021/ja01539a017. Acesso em: 11 ago. 2021.

KOSANOVIĆ, M. M. et al. Electrochemical and Antioxidant Properties of (+)-Catechin, Quercetin and Rutin. Croatica Chemica Acta, v. 83, n. 2, p. 197-207, 2010. Disponível em: https://hrcak.srce.hr/56023. Acesso em: 11 ago. 2021.

LIU, J. et al. Gram-scale production of graphene oxide- $\mathrm{TiO}_{2}$ nanorod composites: Towards high-activity photocatalytic materials. Applied Catalysis B: Environmental, v. 106, n. 1-2, p. 76-82, July 2011. Disponível em: https://www.sciencedirect.com/science/article/pii/ S0926337311002086. Acesso em: 11 ago. 2021.

LOURENÇO, M. A. T. Estudo do mecanismo de eletro-oxidação da morfina. 2004. Trabalho de Conclusão de Curso (Graduação em Engenharia Química) - Universidade Federal de Santa Catarina, Florianópolis, 2004.

MALEKIA, S. J.; CRESPO, J. F.; CABANILLAS, B. Anti-inflammatory effects of flavonoids. Food Chemistry, v. 299, p. 1-11, jul. 2019. Disponível em: https://www.sciencedirect.com/ science/article/pii/S0308814619312300?via\%3Dihub. Acesso em: 11 ago. 2021.

MARRANI, A. G. et al. A. Insights from experiment and theory into the electrochemical reduction mechanism of graphene oxide. Electrochimica Acta, v. 304, p. 231-238, May 2019. Disponível em: https://www.sciencedirect.com/science/article/pii/S0013468619303755. Acesso em: 11 ago. 2021.

MÜLAZIMOĞLU, I. E.; ÖZKAN, E.; SOLAK, A. O. Covalently grafted on to the glassy carbon electrode in non-aqueous media of apigenin and naringenin as different flavonoid derivatives. Analytical \& Bioanalytical Electrochemistry, v. 3, n. 2, p. 102-118, abr. 2011. Disponível em: https://www.researchgate.net/publication/235969018_Covalently_grafted_ onto_the_glassy_carbon_electrode_in_non-aqueous_media_of_Apigenin_and_Naringenin_ as_different_flavonoid_derivatives. Acesso em: 11 ago. 2021. 
MUTHA, R. E.; TATIYA, A. U.; SURANA, S. J. Flavonoids as natural phenolic compounds and their role in therapeutics: an overview. Future Journal of Pharmaceutical Sciences, v. 7, n. 1, p. 7-25, Jan. 2021. Disponível em: https://fjps.springeropen.com/articles/10.1186/s43094020-00161-8? utm_source $=x$ mol\&utm_medium $=$ affiliate $\& u t m \_c o n t e n t=m e t a \& u t m \_$ campaign=DDCN_1_GL01_metadata. Acesso em: 11 ago. 2021.

NOVOSELOV, K. S. et al. Electric field effect in atomically thin carbon films. Science, v. 306, n. 5696, p. 666-669, Oct. 2004. Disponível em: https://www.researchgate.net/publication/284146851_Electric_Field_Effect_in_Atomically_Thin_Carbon_Films. Acesso em: 11 ago. 2021.

PACHECO, W. F. et al. Voltametrias: uma breve revisão sobre os conceitos. Revista Virtual de Quimica, v. 5, n. 4, p. 516-537, ago. 2013. Disponível em: http://rvq-sub.sbq.org.br/index. php/rvq/article/view/380/345. Acesso em: 11 ago. 2021.

REIS, N. S. et al. Métodos eletroquímicos usados para avaliação da atividade antioxidante de produtos naturais. Latin American Journal of Pharmacy, v. 28, n. 6, p. 949-953, 2009. Disponível em: http://www.latamjpharm.org/resumenes/28/6/LAJOP_28_6_3_2.pdf. Acesso em: 11 ago. 2021.

SÁ, L. Z. C. M. de. Determinação eletroanalítica e espectrofotométrica da atividade antioxidante de fermentados de jabuticaba e vinhos de diferentes procedências. 2013. Dissertação (Mestrado em Ciências Farmacêuticas) - Faculdade de Farmácia, Universidade Federal de Goiás, Goiânia, 2013. Disponível em: https://repositorio.bc.ufg.br/tede/handle/tede/3359. Acesso em: 11 ago. 2021.

SHAMKHALICHENAR, H.; CHOI, J. W. Review-Non-Enzymatic Hydrogen Peroxide Electrochemical Sensors Based on Reduced Graphene Oxide. Journal of The Electrochemical Society, v. 167, n. 3, p. 037531, Jan. 2020. Disponível em: https://iopscience.iop.org/article/10.1149/1945-7111/ab644a. Acesso em: 11 ago. 2021.

SMITH, E. T. Examination of $\mathrm{n}=2$ reaction mechanisms that reproduce $\mathrm{pH}$-dependent reduction potentials. Analytica Chimica Acta, v. 572, n. 2, p. 259-264, July 2006. Disponível em: https://www.sciencedirect.com/science/article/pii/S0003267006009949?casa_token=sSnEP1 XgfKIAAAAA:7rj1ZYdcqCQKO3k4ZHNtUC2BYFOvIGl0ZG-ymrxMM9-kV9YhLLf9s NsdjDpE_dmGpl8vX9DoyX4. Acesso em: 12 ago. 2021.

SOKOLOVÁ, R. et al. The oxidation of natural flavonoid quercetin. Chemical Communications, v. 48, n. 28, p. 3433-3435, Apr. 2012. Disponível em: https://www.researchgate.net/ publication/221854869_The_oxidation_of_natural_flavonoid_quercetin. Acesso em: 12 ago. 2021.

TIMBOLA, A. K. et al. Electrochemical Oxidation of Quercetin in Hydro-Alcoholic Solution. Journal of the Brazilian Chemical Society, v. 17, n. 1, p. 139-148, Feb. 2006. Disponível em: https://www.scielo.br/j/jbchs/a/hxFqDxFXKV8TFMfxKg6sGpj/?lang=en. Acesso em: 12 ago. 2021.

TOH, S. Y. et al. Graphene production via electrochemical reduction of graphene oxide: Synthesis and characterization. Chemical Engineering Journal, v. 251, n. 1, p. 422-434, Sept. 2014. Disponível em: https://www.sciencedirect.com/science/article/pii/S138589471400432X?casa_ token=eMsLcRKRHb0AAAAA:iFvNbnXk15F4Aa89CLteZ7jzmOvPPbnG1Aj_ _D_IrvdF116jTo_ffNgwsMxXKrtr6tU70uX2mIg. Acesso em: 12 ago. 2021. 
WANG, J. et al. Comparative studies on electrochemical activity of graphene nanosheets and carbon nanotubes. Electrochemistry Communications, v. 11, n. 10, p. 1892-1895, Oct. 2009. Disponível em: https://www.sciencedirect.com/science/article/pii/S1388248109003828?casa_ token=6yjpxHyGkZoAAAAA:OfIGxUEtH5PNXsEl3rbEQyVPAM6oNnSVIWawK3_0r98x BdjUDtfFael80XP07hiqm6Iu42LYE4Y. Acesso em: 12 ago. 2021.

ZHANG. D. et al. Analysis of the Antioxidant Capacities of Flavonoids under Different Spectrophotometric Assays Using Cyclic Voltammetry and Density Functional Theory. Journal of Agricultural and Food Chemistry, v. 59, n. 18, p. 10277-10285, Aug. 2011. Disponível em: https://www.researchgate.net/publication/51557495_Analysis_of_the_Antioxidant_Capacities_ of_Flavonoids_under_Different_Spectrophotometric_Assays_Using_Cyclic_Voltammetry_ and_Density_Functional_Theory. Acesso em: 12 ago. 2021.

\section{Contato}

Grazielle Santos Castro

graziellecastro17@yahoo.com

\section{Tramitação}

Recebido em abril de 2020.

Aprovado em junho de 2021. 